\title{
Exact properties of the chemical potential-density relation at finite temperature in the Hubbard model *
}

\author{
Sze-Shiang Feng ${ }^{\Uparrow}$, Ferdinando Mancini ${ }^{\S}$ \\ Ф.CCAST(World Lab.), P.O. Box 8730, Beijing 100080 \\ T.Department of Astronomy and Applied Physics \\ University of Science and Technology of China, 230026, Hefei, China \\ e-mail:sshfeng@yahoo.com \\ §. Università degli Studi di Salerno-Unità IFNM di Salerno \\ Dipartmento di Scienze Fisiche "E.R. Caianiello" 84081 Baronissi Salerno, Italy \\ email: mancini@physics.unisa.it
}

November 20, 2018

\begin{abstract}
We draw some rigorous conclusions about the functional properties of the $\mu-\rho$ relation in the Hubbard model based on symmetry considerations and unitary transformations. It is shown that the charge susceptibility reaches its local extreme at half-filling. Exact expressions are obtained in two limiting cases.

PACS number(s): 75.10.Lp,71.20.Ad,74.65.+n,74.20.Mn
\end{abstract}

Key words: $\mu-\rho$ relation,Hubbard model

${ }^{*}$ S.S. Feng is on leave of absence from the Physics Department, Shanghai University, 201800, Shanghai, China 


\section{Introduction}

Exact results are of great interests in discussions of strong correlations [1]. The important one-dimensional strongly correlated models such as Hubbard model and Kondo model can be exactly solved by means of Bethe ansatz 2] 3]. Nevertheless, exact knowledge of higher dimensional models are very rare. Remarkably, the $\eta$-paring mechanism [1] can be employed to discuss the possible existence of the off-diagonal long range order(ODLRO) [5] at both zero and finite temperatures [6] [7]. The exact knowledge about the chemical-potential relation and magnetization, which are important aspects of the statistics, is still called for. Because of this situation, a number of approximate approaches are applied, as well as computational simulations. It should be realized that every reliable approximation must coincide with the exact one qualitatively. That is, it should have the properties of the exact solution. Therefore, the exact properties of the $\mu-\rho$ relation should be studied. This is the motivation of this paper. The layout of this paper is as follows. Section 2 discusses the general exact properties of $\mu-\rho$ relation. The last section is devoted to conclusional remarkes.

\section{Properties of the $\mu-\rho$ relation}

The Hamiltonian of the generic Hubbard model on lattice $\Lambda$ is

$$
H=\sum_{i j} \sum_{\sigma} t_{i j} c_{i \sigma}^{\dagger} c_{j \sigma}+U \sum_{i} n_{i \uparrow} n_{i \downarrow}
$$

and the grand canonical partition function is

$$
Z(\mu, \beta ; t, U)=\operatorname{Tr} \exp [-\beta(H-\mu N)]
$$

where $c_{i \sigma}^{\dagger}$ and $c_{i \sigma}$ are the creation and annihilation operators of the electrons with spin $\sigma=\uparrow, \downarrow$ at site $i$. The hopping matrix $\left\{t_{i j}\right\}$ is required to be real and symmetric. The number operators

are $n_{i \sigma}=c_{i \sigma}^{\dagger} c_{i \sigma}$, while the $U$ denotes the on-site Coulomb interaction. It is further assumed that the the lattice $\Lambda$ is bipartite in the sense that it can be divided into sublattices $\mathbf{A}$ and B such that $t_{i j}=0$ whenever $\{i j\} \in \mathbf{A}$ or $\{i j\} \in \mathbf{B} . N=\sum_{i, \sigma} n_{i \sigma}$ is the number operator of the electrons. Though the desired $\mu-\rho$ relation is of the form $\mu=\mu(\rho, \beta)$ in statistics, the properties to be discussed below is that of the inverse relation $\rho=\rho(\mu, \beta)$. 
We first derive a recursion relation concerning the moments of the number operators. For this purpose, we make use of the complete particle-hole transformation

$$
P c_{i \sigma} P^{-1}=\epsilon(i) c_{i \sigma}^{\dagger}
$$

where $\epsilon(i)=1 /-1$ if $i \in \mathbf{A} / \mathbf{B}$. Under this transformation, the grand canonical Hamiltonian undergoes the following transformation

$$
P(H-\mu N) P^{-1}=H+U\left(N_{\Lambda}-N\right)-\mu\left(2 N_{\Lambda}-N\right)
$$

where $N_{\Lambda}$ is the total number of sites of the lattice $\Lambda$. Therefore, the partition function satisfies the following relation

$$
Z(\mu, \beta ; t, U)=\exp \left[-\beta(U-2 \mu) N_{\Lambda}\right] Z(U-\mu, \beta ; t, U)
$$

We have accordingly for $n=1,2,3, \cdots$

$$
\begin{aligned}
<N^{n}>_{\mu} & =Z^{-1} \operatorname{Tr} P N^{n} P^{-1} \exp \left[-\beta P\left(H_{0}-\mu N\right) P^{-1}\right] \\
& =Z^{-1} \operatorname{Tr}\left(2 N_{\Lambda}-N\right)^{n} \exp \left[-\beta P\left(H_{0}-\mu N\right) P^{-1}\right] \\
& =<\left(2 N_{\Lambda}-N\right)^{n}>_{U-\mu}
\end{aligned}
$$

This relation holds also locally. Consider the local density operator $n_{i}=\sum_{\sigma} c_{i \sigma}^{\dagger} c_{i \sigma}$, using the transformation (3), the same reasoning leads to

$$
<n_{i}^{k}>_{\mu}=<\left(2-n_{i}\right)^{k}>_{U-\mu}
$$

This local relation can not be obtained from (8) directly. In particular, we have at half-filling for which $\mu=\mu_{c}=U / 2$ from $(9)<n_{i}>_{\mu_{c}}=1$. The global relation (8) gives then

$$
<N^{n}>_{\mu_{c}}=<\left(2 N_{\Lambda}-N\right)^{n}>_{\mu_{c}}
$$

That is

$$
\left[1+(-1)^{n+1}\right]<N^{n}>_{\mu_{c}}=\sum_{l=1}^{n} C_{n}^{l}\left(2 N_{\Lambda}\right)^{l}(-1)^{n-l}<N^{n-l}>_{\mu_{c}}
$$

where $C_{n}^{l}$ is the combinatorial number: $C_{n}^{l}=\frac{n !}{l !(n-l) !}$. The first three cases for odd $n$ are

$$
\begin{aligned}
<N>_{\mu_{c}} & =N_{\Lambda} \\
<N^{3}>_{\mu_{c}} & =3 N_{\Lambda}<N^{2}>_{\mu_{c}}-2 N_{\Lambda}^{3} \\
<N^{5}>_{\mu_{c}} & =5 N_{\Lambda}<N^{4}>_{\mu_{c}}-20 N_{\Lambda}^{3}<N^{2}>_{\mu_{c}}+16 N_{\Lambda}^{5}
\end{aligned}
$$


In general, eq.(10) turn out to be identities for even $n$ and it provides non-trivial information only for odd $n$. On the other hand, since the fluctuation

$$
\frac{1}{\beta} \frac{\partial}{\partial \mu}<N>_{\mu}=<N^{2}>_{\mu}-<N>_{\mu}^{2}
$$

is of order $\langle N\rangle$, we may introduce a function $\alpha$ via

$$
\frac{1}{\beta} \frac{\partial}{\partial \mu}<N>_{\mu}=\alpha(\mu)<N>_{\mu}
$$

One can see that the derivatives of $\langle N\rangle$ with respect to $\mu$ exist at every order. Obviously, one has

$$
\alpha \geq 0
$$

The function $\alpha$ is related to the compressibility which is defined by

$$
\kappa=-\frac{1}{V}\left(\frac{\partial V}{\partial P}\right)_{T, N}
$$

as follows. Since $\rho=N / V$, we may express $\kappa$ as

$$
\kappa=\frac{1}{\rho}\left(\frac{\partial \rho}{\partial P}\right)_{T, N}=\frac{1}{\rho}\left(\frac{\partial \rho}{\partial \mu}\right)_{T, N}\left(\frac{\partial \mu}{\partial P}\right)_{T, N}
$$

Using the thermodynamic relations $\$$

$$
\mu=\left(\frac{\partial \Phi}{\partial N}\right)_{P, T}, \quad V=\left(\frac{\partial \Phi}{\partial P}\right)_{T, N}
$$

where $\Phi$ is the thermodynamic potential, we have

$$
\left(\frac{\partial \mu}{\partial P}\right)_{T, N}=\left(\frac{\partial V}{\partial N}\right)_{T, P}=\frac{1}{\rho}
$$

therefore

$$
\kappa=\frac{1}{\rho^{2}}\left(\frac{\partial \rho}{\partial \mu}\right)_{T}=\frac{\alpha \beta}{\rho}
$$

This relation is more thermodynamical than statistical. It holds for both fermi liquids and non-fermi liquids. For the electron gas at zero temperature, this relation can be derived in another way [9]. In the Hubbard model, the density is defined by $\rho=\langle N\rangle / N_{\Lambda}$. Since $\langle N\rangle$ has maximum value $2 N_{\Lambda}$ and is a monotonically increasing function of $\mu$, we have

$$
\lim _{\rho \rightarrow 2} \alpha=0
$$


We now prove some more properties of $\alpha$. Firstly, for a homogeneous system, we have $<N>=N_{\Lambda}<n_{i}>$ and $<N^{2}>=\sum_{i, j}<n_{i} n_{j}>=N_{\Lambda} \sum_{i}<n_{i} n_{0}>$. Thus (15) implies that

$$
\kappa=\frac{\beta}{\rho^{2}} N_{\Lambda}\left[\frac{1}{N_{\Lambda}} \sum_{i}<n_{i} n_{0}>-<n_{0}>^{2}\right]
$$

which provides another way to evaluate the compressibility. Define the density-density correlation function

$$
C_{i \sigma, j \sigma^{\prime}}(\mu)=<n_{i \sigma} n_{j \sigma^{\prime}}>-<n_{i \sigma}><n_{j \sigma^{\prime}}>
$$

the relation

$$
C_{i \sigma, j \sigma^{\prime}}(\mu)=C_{i \sigma, j \sigma^{\prime}}(U-\mu)
$$

can be obtained (not only for a homogeneous system) by the local relation(9). Secondly, applying the complete particle-hole transformation to eq.(9), we have

$$
<\left(2 N_{\Lambda}-N\right)^{2}>_{U-\mu}-<2 N_{\Lambda}-N>_{U-\mu}^{2}=\alpha(\mu)<2 N_{\Lambda}-N>_{U-\mu}
$$

Expanding this relation and using eq(15) for $\mu \rightarrow U-\mu$, one can obtain

$$
[\alpha(\mu)+\alpha(U-\mu)]<N>_{U-\mu}=\alpha(\mu) 2 N_{\Lambda}
$$

Thirdly, differentiate (28) with respect to $\mu$, we have

$$
\left[\alpha^{\prime}(\mu)+\alpha^{\prime}(\nu)(-1)_{\mid \nu=U-\mu}\right]<N>_{U-\mu}+[\alpha(\mu)+\alpha(U-\mu)] \frac{\partial}{\partial \nu}<N>_{\nu}(-1)_{\mid \nu=U-\mu}=\alpha^{\prime}(\mu) 2 N_{\Lambda}
$$

Therefore, at $\mu=\mu_{c}$

$$
2 \alpha\left(\mu_{c}\right)(-1) \frac{\partial}{\partial \mu}<N>_{\mid \mu_{c}}=\alpha^{\prime}\left(\mu_{c}\right) 2 N_{\Lambda}
$$

Hence we obtain another relation

$$
\alpha^{\prime}\left(\mu_{c}\right)=-\beta \alpha^{2}\left(\mu_{c}\right)
$$

Using this relation, one can prove that the charge susceptibility $\chi_{c}:=\frac{\partial \rho}{\partial \mu}$ takes its extreme at half-filling, i,e,

$$
\chi_{c}^{\prime}\left(\mu_{c}\right)=0
$$

This is a rigorous result and is valid in all cases at finite temperature. Unfortunately, we can not know whether it is a minimum or a maximum at the present time. If it is a minimum and 
the value $\chi_{c}\left(\mu_{c}\right)=0$, the system will demonstrate a Mott transition.

We next study the Taylor expansion of $\rho=\rho(\mu)$ around $\mu=\mu_{c}$. From eq(8) we have

$$
\rho(\mu)=2-\rho(U-\mu)
$$

If we define

$$
f(\mu)=\rho\left(\mu_{c}+\mu\right)-1
$$

then

$$
f(\mu)=-f(-\mu)
$$

i.e. $f(\mu)$ is an odd function of $\mu$. For finite temperature, $\rho=\rho(\mu)$ is an analytic function and can be expanded in powers of $\mu-\mu_{c}$, thereby, we may infer that the Taylor expansion of $\rho(\mu)$ must be of the form at finite temperature

$$
\rho(\mu)=1+\sum_{k=0}^{\infty} \frac{\rho^{(2 k+1)}\left(\mu_{c}\right)}{(2 k+1) !}\left(\mu-\mu_{c}\right)^{2 k+1}
$$

The average values $\left\langle N^{n}\right\rangle_{\mu_{c}}$ for both odd and even $n$ must be known in order to know the Taylor coefficients in (36). Unfortunately, the recursion relation eq(8) can not provide us with useful information about $\left\langle N^{n}>_{\mu_{c}}\right.$ for even $n$. Otherwise, the exact form of $\mu-\rho$ relation can be immediately obtained after the sum is implemented. In any case, equation of motion is needed in order to calculate $\left\langle N^{k}>\right.$ for the moment.

Though the general exact $\mu-\rho$ relation is not accessible so far, the specific expressions for two limiting cases: non-interacting case and atomic case are obtainable nevertheless. In the first case, $U=0$, if the lattice is periodic, we can work in the $\mathbf{k}$-space and we have

$$
<n_{\mathbf{k} \sigma}>=\frac{1}{e^{\beta[t(\mathbf{k})-\mu]}+1}
$$

where $t(\mathbf{k})$ is the Fourier transform of $t_{i j}$. Therefore the $\mu-\rho$ relation is

$$
\rho=\frac{1}{N_{\Lambda}} \sum_{\mathbf{k} \sigma}<n_{\mathbf{k} \sigma}>
$$

In the second case, $t_{i j}=0$, the partition function can be calculated

$$
Z(\beta, \mu ; U)=\operatorname{Tr} \exp \left[-\beta\left(U \sum_{i} n_{i \uparrow} n_{i \downarrow}-\mu N\right)\right]=\left[1+2 e^{\beta \mu}+e^{\beta(2 \mu-U)}\right]^{N_{\Lambda}}
$$

Therefore, the average density is

$$
\rho(\mu, \beta)=\frac{2 e^{\beta\left(\mu-\mu_{c}\right)} e^{\beta \mu_{c}}+2 e^{2 \beta\left(\mu-\mu_{c}\right)}}{1+2 e^{\beta\left(\mu-\mu_{c}\right)} e^{\beta \mu_{c}}+e^{2 \beta\left(\mu-\mu_{c}\right)}}
$$


This relation is also exact for the case of infinite hopping range[[10]. It can be seen that the Taylor coefficients must be in general both $t_{i j}$ and $U$ dependent as can be seen from the two special cases (38) and (40). The inverse relation at finite temperature $\mu=\mu(\rho, \beta)$ can be obtained. For $\rho \neq 2$ we have

$$
\mu=\frac{1}{\beta} \ln \left[\rho-1+\sqrt{(\rho-1)^{2}+\rho(2-\rho) e^{-2 \beta \mu_{c}}}\right]-\frac{1}{\beta} \ln (2-\rho)+2 \mu_{c}
$$

while for $\rho=2$, we can obtain only $\mu=\infty$ at finite temperature otherwise the denorminator in (40) is finite and can be multiplied by both sides and in this way we will reach the unacceptable conclusion $e^{\beta \mu}=-1$. It should be noted that discontinuities may appear at zero temperature. For instance, if $\mu_{c}>0$ we have from (40)

$$
\lim _{\beta \rightarrow \infty} \mu= \begin{cases}\lim _{\beta \rightarrow \infty} \frac{1}{\beta} \ln \left[\frac{\rho(2-\rho)}{2|\rho-1|} e^{-2 \beta \mu_{c}}\right]+2 \mu_{c}=0 & \text { if } \rho<1 \\ \mu_{c} & \text { if } \rho=1 \\ 2 \mu_{c} & \text { if } \rho>1\end{cases}
$$

while for $\mu_{c}<0$ and $\rho \neq 2, \lim _{\beta \rightarrow \infty} \mu=\mu_{c}$.

For $t_{i j} \neq 0$, numerical and analytical results show that there is a critical value $U_{c}$ such that for $U>U_{c}$ at $T=0$, there is a gap in the density of states and a discontinuity of $\mu[11]$.

Introducing the dimensionless parametres $\tilde{t}=\beta t, \tilde{\mu}=\beta \mu, \tilde{U}=\beta U, \rho$ is a function of these parametres: $\rho=\rho(\tilde{t}, \tilde{\mu}, \tilde{U})$. Since under the Lieb-Wu transform in [2], $t \rightarrow-t$, the dependence of $t$ is in fact through $t^{2}$. Hence we have

$$
\rho(\beta, \mu ; t, U)=\rho(\tilde{\mu} ; \tilde{t}, \tilde{U})=1+\sum_{k=0}^{\infty} \frac{\rho^{(2 k+1)}\left(\tilde{\mu}_{c} ; \tilde{t}^{2}, \tilde{U}\right)}{(2 k+1) !}\left(\tilde{\mu}-\tilde{\mu}_{c}\right)^{2 k+1}
$$

\section{Discussions}

We discussed exact properties of the chemical potential-density relation by means of the particle-hole transformations and the symmetries of the Hubbard model. Similar discussion applies to Kondo lattice model and periodic Anderson models as in [7]. These exact features can help us to evaluate the reliabilities of the various conclusions from different approximations. Yet, these properties are still not enough to determine the relations completely. It can be inferred from the exact solutions in the two limiting cases that the general exact answer must be extremely complicated. 
AcknoledgementThis work is supported by the Funds for Young Teachers of Shanghai Education Committee, the National Science Foundation of China under Grant.No. 19805004 and by Instituto Nazionale di Fisica Della Materia, Unità di Salerno .

\section{References}

[1] V.E. Korepin \& F.H.Eßler. Eds. Exact Sovable Models of Strongly Correlated Electrons (World Scientific, Singapore, 1994).

[2] E.H. Lieb \& F.Y Wu Phys. Rev. Lett.20(1968):1445.

[3] N. Andrei Phys. Rev. Lett.45 (1980):379. P.B. WiegmannJETP Lett.31 (1980)364.

[4] C.N. Yang Phys. Rev. Lett.63 (1989):2144.

[5] C.N. Yang Rev. Mod. Phys. 34 (1962):694.

[6] C.N. Yang \& S.C. Zhang Mod. Phys. Lett.B4 (1990):759; C.N. Yang Phys. Lett. A161 (1991):292.

[7] S.S. Feng Mod. Phys. Lett. B12 (1998):555; S.S. Feng cond-mat/9902113 Phys. Lett. A256 (1999):230

[8] L.D. Landau \& E.M. Lifshitz Statistical Physics part I (Pergamon Press, N.Y.,1986).

[9] G.D. Mahan Many-Particle Physics (Plenum Press, NY. 1981). Sec.5.6.

[10] P.van Dongen \& D. Vollhadt Phys. Rev. B40 (1989):7252;

[11] F. Mancini The Mott-Hubbard transition and the paramagnetic insulating state in the two-dimensional Hubbard model .cond-mat/9812310 